\title{
Treatment of gastrointestinal stromal tumor with liver metastasis by surgery combined with imatinib in a male patient: a case report
}

\author{
Wenjun Xiong", Haipeng Huang", Yuling Xue, Jin Li, Jin Wan, Wei Wang \\ Department of Gastrointestinal Surgery, Guangdong Provincial Hospital of Chinese Medicine, the Second Affiliated Hospital of Guangzhou \\ University of Chinese Medicine, Guangzhou, China \\ "These authors contributed equally to this work. \\ Correspondence to: Wei Wang, MD, PhD. Department of Gastrointestinal Surgery, Guangdong Provincial Hospital of Chinese Medicine, Dade Road \\ No. 111, 510120, Guangzhou, China. Email: wangwei16400@163.com.
}

\begin{abstract}
Gastrointestinal stromal tumors (GIST) are the most common mesenchymal tumors of the gastrointestinal tract and liver are the main sites of metastasis. At present, no uniform treatment standard but surgery combined with imatinib is recommended for GIST with potentially resectable liver metastases. A 77 years old male patient was diagnosed as giant duodenal GIST with multiple liver metastases. After 10 months of oral imatinib treatment from February 2015 to December 2015, the tumor lesions were evaluated as partial response and converted into resectable lesions. Then the patient successfully underwent R0 surgical excision and continued to take imatinib after the operation. The patient recovered uneventfully and no tumor recurrence and metastasis were found 2 years postoperatively. This case report has shown that surgery combined with targeted treatment is effective for the treatment of GIST with liver metastases. Surgery combined with oral imatinib is the first choice for the treatment of GIST with liver metastases because it could transform an unresectable lesion into a resectable one. This case report has shown that surgery combined with targeted treatment is effective for the treatment of GIST with multiple liver metastases.
\end{abstract}

Keywords: Gastrointestinal stromal tumor (GIST); liver metastases; surgical treatment; targeted treatment; case report

Received: 21 June 2020; Accepted: 01 August 2020; Published: 10 November 2020.

doi: 10.21037 /gist-19-4

View this article at: http://dx.doi.org/10.21037/gist-19-4

\section{Introduction}

GIST is the most common mesenchymal tumor of gastrointestinal tract, accounting for $1-3 \%$ of gastrointestinal malignant tumor. Liver is the major metastatic site of GIST in spite of before or after surgery $(1,2)$. With the further understanding of the biological characteristics of GIST, it is generally believed that there is no absolute differentiation between benign and malignant GIST. Benign GIST also has long-term metastasis risk (3). Immunohistochemical antibody CD117, DOG-1, CD34 and so on are helpful for differential diagnosis (4). Surgical excision was the only treatment for GIST before imatinib was used as a targeted drug in the treatment of GIST.
Imatinib, a molecular targeting tyrosine kinase inhibitor, has become a first-line drug of GIST (5). Today, the treatment of GIST has changed from simple surgical excision to surgical excision combined with targeted drug therapy, especially for the patients with liver metastasis. Here we report 1 case of GIST patient diagnosed and treated in our hospital. At the onset, the patient was unsuitable for surgery because of the giant duodenal GIST with multiple liver metastases. After targeted therapy with imatinib, the tumors converted into resectable lesions and was successfully performed $\mathrm{R} 0$ resection, and the patient was confirmed to be cancer-free survival for more than two years after surgery. We present the following case in accordance with 


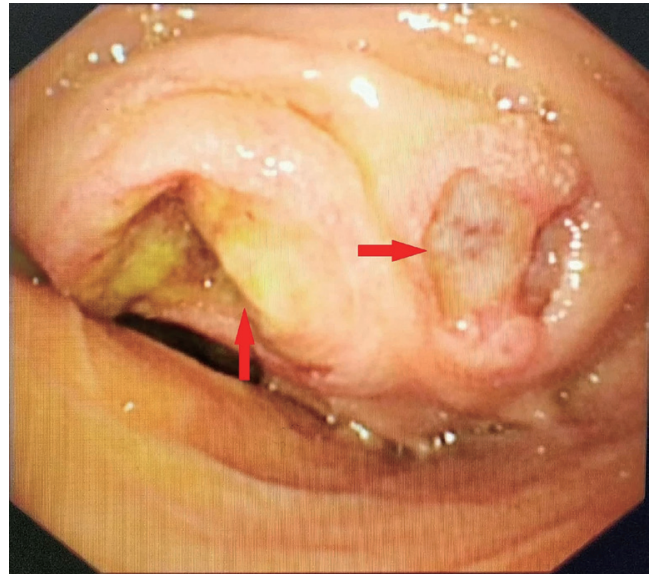

Figure 1 2015-02-18 electronic gastroscopy: descendent duodenal mass and ulcer. Arrows: the location of the tumor.

the CARE reporting checklist (available at http://dx.doi. org/10.21037/gist-19-4).

\section{Case presentation}

All procedures performed in studies involving human participants were in accordance with the ethical standards of the institutional and/or national research committee(s) and with the Helsinki Declaration (as revised in 2013). Written informed consent was obtained from the patient.

\section{Chief complaints}

A 77 years old male patient has been hospitalized for repeated black stool for 5 years, as well as wasting and fatigue for more than 1 year.

\section{History of present illness}

Five years ago, the patient began to appear black stool (About $200 \mathrm{~mL}$ at a time) without obvious incentive, and no accompanying abdominal pain, abdominal distention, nausea and hematemesis, and didn't accept special treatment at that time. His highest weight was $65 \mathrm{~kg}$ and gradually decreased to $52.5 \mathrm{~kg}$ in one year from 2014 to 2015, accompanied by systemic fatigue, physical decline, occasional upper abdominal pain.

\section{History of past illness}

The patient had a free previous medical history.

\section{Physical examination}

The patient's vital signs were stable. There was no tenderness or rebound pain in the abdomen. A mass was touched in the right middle abdomen with a diameter of about $7 \mathrm{~cm}$, smooth surface, poor mobility, and no tenderness.

\section{Laboratory and imaging examinations}

Laboratory examination indicated a red blood cell count of $3.07 \times 10^{12} / \mathrm{L}$, a hemoglobin level of $56 \mathrm{~g} / \mathrm{L}$. Fecal occult blood test was positive (2+).

2015-02-18 electronic gastroscopy: descendent duodenal mass and ulcer. Puncture biopsy of duodenal mass showed that was consistent with gastrointestinal stromal tumors (Figure 1). 2015-03-03 Abdominal computed tomography (CT): (I) Giant mass is in the right middle abdomen and considered as duodenal descending mesenchymal malignant tumor. (II) Multiple metastases in the liver (Figure 2A). The results of gene detection in duodenal lesions revealed positive mutation for exon 11, while negative mutation for exon 9, 10, 13 of KIT gene.

\section{Final diagnosis}

The patient was diagnosed with giant duodenal GIST with multiple liver metastases and severe anemia.

\section{Treatment}

Considering giant duodenal GIST and multiple liver metastases, the patient was first treated by imatinib $400 \mathrm{mg} / \mathrm{d}$ according to the results of gene testing $(6,7)$ and periodic reviewed (Every 3-4 months). After 10 months of oral imatinib treatment from February 2015 to December 2015 , the tumor lesion was evaluated as partial response according to CT results (Figure 2B,C,D) and patient's symptoms. In January 2016, after multi-disciplinary treatment (MDT) discussion and excluding surgical contraindications, we performed duodenal stromal tumor resection + right hemicolectomy + partial enterectomy + partial hepatectomy (left lateral lobe tumor resection) + jejuno-duodenal anastomosis (Roux-en-y anastomosis) under general anesthesia with endotracheal intubation. Postoperative pathological diagnosis: (duodenal stromal tumor + right colon + partial small intestine) Tumor was $8 \mathrm{~cm} \times 7 \mathrm{~cm} \times 6 \mathrm{~cm}$ in size, single lesion, nuclear fission 

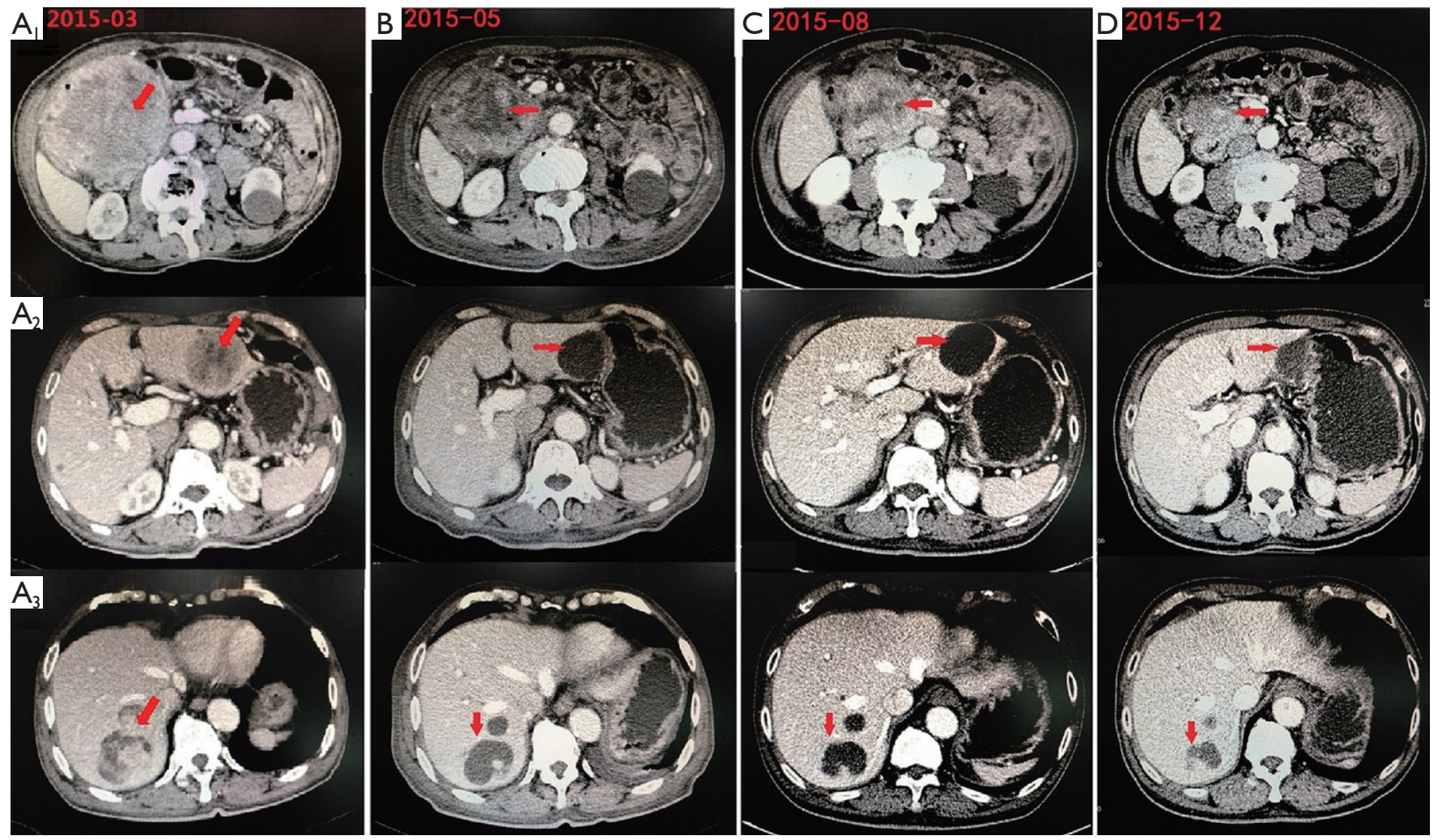

Figure $2\left(\mathrm{~A}_{1}\right)$ 2015-03 Abdominal computed tomography (CT): Giant mass (approximately $13 \mathrm{~cm} \times 12 \mathrm{~cm}$ in size) in the right middle abdomen. It is considered as duodenal descending mesenchymal malignant tumor. $\left(\mathrm{A}_{2}, \mathrm{~A}_{3}\right)$ Multiple metastases in the liver. (B,C,D) 2015-5 to 2015-12 Abdominal CT: After 10 months of imatinib treatment, CT scan images show that the mass in descending duodenum were smaller (about $6.7 \mathrm{~cm} \times 6.7 \mathrm{~cm}$ in size) than before, and necrosis and cystic degeneration inside lesion were obvious. Nodules of hepatic metastasis were also partly smaller than before. Arrows: the location of the lesions.

$\leq 5 / 50 \mathrm{HPF}$, negative margin was and no lymph node metastasis (Figure 3). Immunohistochemical analysis: CD34 (+), CD117 (+), DOG-1 (+), SMA (+), Desmin (-), Ki-67 (positive expression rate is about $2 \%$ ). It was consistent with GIST. (liver) Nodular mass was $4 \mathrm{~cm}$ in diameter with gelatinous section. Edema and necrosis of tissue and a small number of spindle cells were observed under microscopy. Combined with clinical manifestation, it was considered to be a change in liver metastases of GIST after imatinib therapy (Figure 4). One week after the operation, the patient was continuously and regularly treated with imatinib (400 mg/d).

\section{Outcome and follow-up}

The patient recovered well when discharged from the hospital without complaining of special discomfort, and the abdominal incision healed well. The patients have been followed-up for more than 2 years after operation, and no tumor recurrence and metastasis were found (Figure 5).

\section{Discussion}

GISTs are the most common gastrointestinal tract tumor originating from mesenchymal tissue. The tumor has unique morphological characteristics, mainly manifested as CD117(c-kit) positive and activated by c-kit or PDGFRA gene mutation (8). GIST are most common in the stomach, or even a few cases of multiple metastases without primary lesions. Although GIST tend to be malignant, they rarely invade lymph nodes, so the overall goal of surgical treatment is to achieve $\mathrm{R} 0$ resection without the need for preventive or systematic lymph node dissection (9). Surgery was once the only curable method for GIST. Subsequently, the pathogenesis and targeted drugs of GISTs were gradually broken down by researchers. 


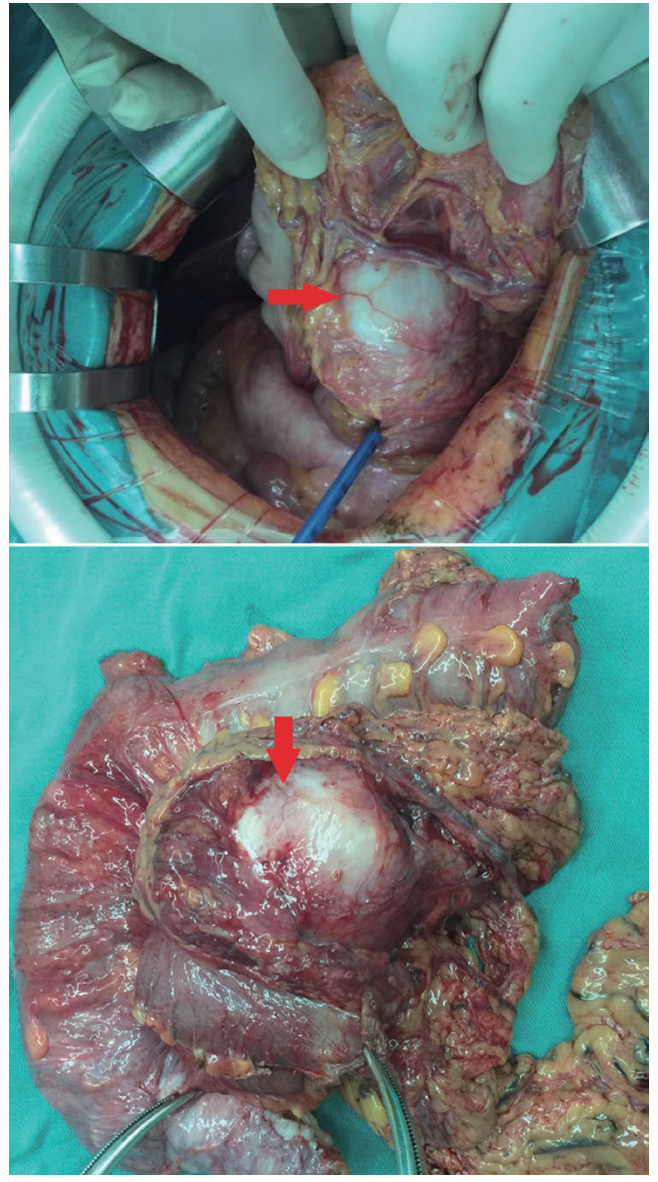

Figure 3 Postoperative specimen (duodenal stromal tumor + right colon + partial small intestine). Postoperative pathological diagnosis: Tumor was $8 \mathrm{~cm} \times 7 \mathrm{~cm} \times 6 \mathrm{~cm}$ in size, single lesion, nuclear fission $\leq 5 / 50 \mathrm{HPF}$. Incision margin was negative and no lymph node metastasis. It was consistent with GIST. Arrow: the location of the tumor.

Currently, the main treatments for GISTs include surgical resection and targeted drugs.

Imatinib is the main targeted drug and become the standard for the treatment of GIST. It is a small molecule tyrosine kinase inhibitors (TKIs), targeting c-kit, PDGFRA and bcr-abl genes. The efficiency can reach $40 \%$ to $50 \%$ for the treatment of patients with unresectable or metastatic GIST (10). The efficiency can reach $40 \%$ to $50 \%$. Studies have shown that patients with advanced GIST could survive for five years, or even longer after taking imatinib (11).

Treatment of GIST with liver metastases also includes surgical resection and targeted therapy. At present, there is no unified criterion for surgical indication of liver

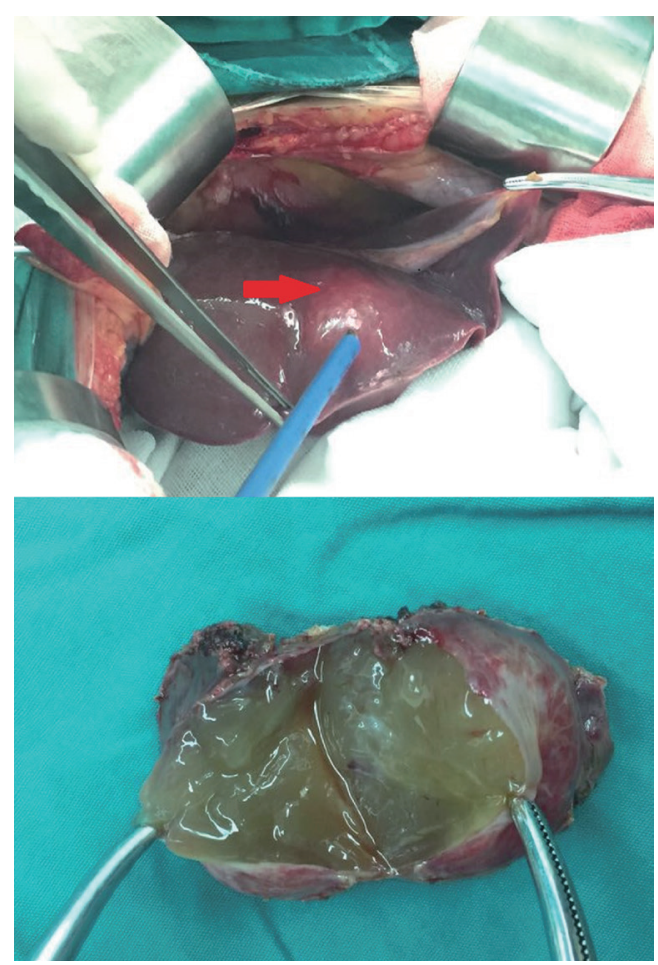

Figure 4 Postoperative specimen (left liver). Pathological diagnosis: nodular mass was $4 \mathrm{~cm}$ in diameter with gelatinous section. Edema and necrosis of tissue and a small number of spindle cells were observed under microscopy. It was considered to be a change in liver metastases of GIST after taking imatinib. Arrow: the location of the tumor.

metastasis. According to the experience of liver metastasis from colorectal cancer, surgical resection can be considered as long as the liver function is well-behaved, the general situation is tolerable, the liver metastases can be completely resected, and the residual liver volume can maintain the basic function (12). For patients with large tumors who can not be operated immediately, imatinib can be used first. Radiofrequency ablation, interventional therapy and liver transplantation can also be used as one of the options. Studies have also shown that imatinib should be used in the treatment of patients with liver metastases, regardless of whether they are operated or not (13). However, more than half of the patients developed drug resistance after about two years of taking imatinib (14).

According to the U.S. National Comprehensive Cancer Network (NCCN) and consensus on diagnosis and treatment for GIST of China $(6,7)$, the targeted therapy is recommendable for the GIST of liver metastasis patients. In 


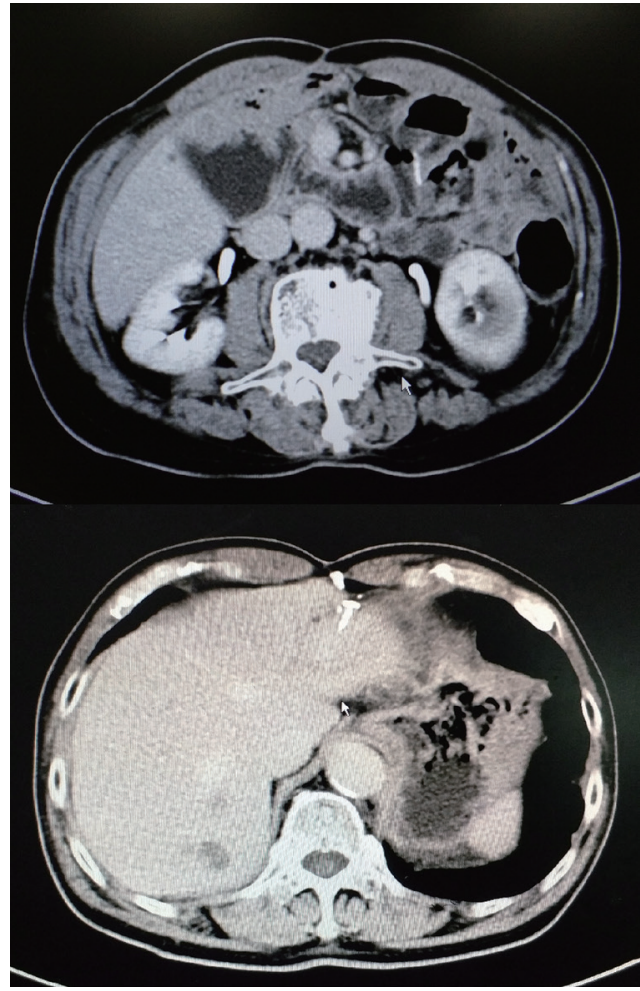

Figure 5 2018-05 Abdominal CT (2 years after operation): no obvious thickening of the jejunal-duodenal anastomotic stoma was observed, and no signs of tumor recurrence. The left liver was postoperative changes and no tumor lesion exit.

this case, imatinib was given to the patient before operation and periodic review was took to evaluate efficacy. Surgical resection was actively evaluated to achieve R0 resection when tumors achieved partial response. Then the patients underwent surgical excision successfully and continued to take imatinib 2 weeks after the operation. The patients have been followed up until now for more than 2 years, and no tumor recurrence and metastasis were found.

\section{Conclusions}

This case report has shown that surgery combined with targeted treatment is effective for the treatment of GIST with liver metastases. Liver metastasis is common in GIST. For different patients, how to choose individualized treatment is a challenge for clinicians. Surgery combined with oral imatinib is the first choice for the treatment of GIST with liver metastases because it could transform an unresectable lesion into a resectable one. With regard to postoperative patients, regular review and follow-up can timely detect the recurrence and metastasis and improve the prognosis.

\section{Acknowledgments}

Funding: This work was supported by the grants from the Clinical Research Project of Guangdong Hospital of Traditional Chinese Medicine (No. YN10101911) and Scientific Research Project of Traditional Chinese Medicine Bureau of Guangdong Provincial (No. 2019KT1049).

\section{Footnote}

Reporting Checklist: The authors have completed the CARE reporting checklist. Available at http://dx.doi.org/10.21037/ gist-19-4

Conflicts of Interest: All authors have completed the ICMJE uniform disclosure form (available at http://dx.doi. org/10.21037/gist-19-4). The authors have no conflicts of interest to declare.

Ethical Statement: The authors are accountable for all aspects of the work in ensuring that questions related to the accuracy or integrity of any part of the work are appropriately investigated and resolved. All procedures performed in studies involving human participants were in accordance with the ethical standards of the institutional and/or national research committee(s) and with the Helsinki Declaration (as revised in 2013). Written informed consent was obtained from the patient.

Open Access Statement: This is an Open Access article distributed in accordance with the Creative Commons Attribution-NonCommercial-NoDerivs 4.0 International License (CC BY-NC-ND 4.0), which permits the noncommercial replication and distribution of the article with the strict proviso that no changes or edits are made and the original work is properly cited (including links to both the formal publication through the relevant DOI and the license). See: https://creativecommons.org/licenses/by-nc-nd/4.0/.

\section{References}

1. Katz SC, DeMatteo RP. Gastrointestinal stromal tumors and leiomyosarcomas. J Surg Oncol 2008;97:350-9.

2. Biasco G, Velo D, Angriman I, et al. Gastrointestinal stromal tumors: report of an audit and review of the 
literature. Eur J Cancer Prev 2009;18:106-16.

3. Tivadar B, Serban B, Mircea M, et al. Late hepatic metastasis in the evolution of gastrointestinal stromal tumors. Hepatogastroenterology 2010;57:95-7.

4. Ríos-Moreno MJ, Jaramillo S, Pereira Gallardo S, et al. GastrointestinaI stromal tumors (GISTs): CD117, DOG1 and PKCO expression. Is there any advantage in using several markers7. Pathol Res Pract 2012;208:74-81.

5. Li J, Gong FJ, Shen L. Post-operative imatinib in patients with intermediate or high risk gastrointestinal stromal tumor. Euro J Sur Oncol 2011;37:319-24.

6. von Mehren M, Randall RL, Benjamin RS, et al. Soft Tissue Sarcoma, Version 2.2018, NCCN Clinical Practice Guidelines in Oncology. J Natl Compr Canc Netw 2018;16:536-63.

7. Li J, Ye Y, Wang J, et al. Chinese consensus guidelines for diagnosis and management of gastrointestinal stromal tumor. Chin J Cancer Res 2017;29:281-93.

8. Lau S, Tam KF, Kam CK, et al. Imaging of gastrointestinal stromal tumor (GIST). Clin Radiol 2004,59:487-98.

9. Gheorghe M, Predescu D, Iosif C, et al. Clinical and therapeutic considerations of GIST. J Med Life

doi: $10.21037 /$ gist-19-4

Cite this article as: Xiong W, Huang $\mathrm{H}$, Xue Y, Li J, Wan J, Wang W. Treatment of gastrointestinal stromal tumor with liver metastasis by surgery combined with imatinib in a male patient: a case report. Gastrointest Stromal Tumor 2020;3:2.
2014;7:139.

10. Gronchi A, Fiore M, Miselli F, et al. Surgery of residual disease following molecular-targeted therapy with imatinib mesylate in advanced/metastatic GIST. Ann Surg 2007;245:341-6.

11. Kanda T, Ishikawa T, Hirota S, et al. Prospective observational study of imatinib therapy in Japanese patients with advanced gastrointestinal stromal tumors: long-term follow-up and second malignancy. Jpn J Clin Oncol 2012;42:578-85.

12. Seesing MF, Tielen R, van Hillegersberg $\mathrm{R}$, et al. Resection of liver metastases in patients with gastrointestinal stromal tumors in the imatinib era: A nationwide retrospective study. Eur J Surg Oncol 2016;42:1407-13.

13. Ye YJ, Gao ZD, Poston GJ, et al. Diagnosis and multidisciplinary management of hepatic metastases from gastrointestinal stromal tumor (GIST). Eur J Surg Oncol 2009;35:787-92.

14. Braconi C, Bracci R, Cellerino R. Molecular targets in Gastrointestinal Stromal Tumors (GIST) therapy. Curr Cancer Drug Targets 2008;8:359-66. 